\title{
Management of Persistent Pediatric Cervical Lymphadenopathy
}

\author{
Katherine C. Wai, MD; ; Tammy J. Wang, MD; Edward Lee, MD; Kristina W. Rosbe, MD \\ Department of Otolaryngology-Head and Neck Surgery, University of California San Francisco, \\ San Francisco, California, USA
}

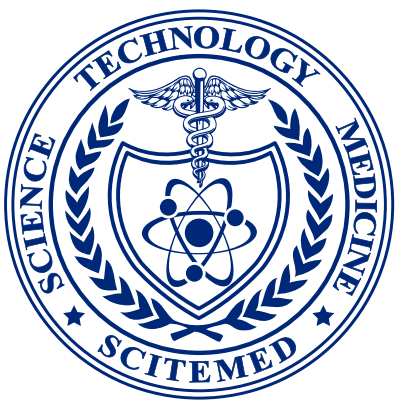

\begin{abstract}
Objective: Asymptomatic cervical lymphadenopathy is a common referral diagnosis for pediatric otolaryngologists in the outpatient setting. Parents and referring care providers are often highly anxious about the risk of lymphoma. Definitive diagnosis requires excisional biopsy under general anesthesia but is rarely indicated and is associated with small but real risks. Current literature provides little guidance on less invasive monitoring. The aim of the study was to characterize children with asymptomatic cervical lymphadenopathy including natural history, radiologic and pathologic findings, and provide guidance in diagnostic and therapeutic intervention and follow-up.

Methods: A retrospective cohort study was performed to identify patients $<18$ years old referred to outpatient pediatric otolaryngology and general surgery for cervical lymphadenopathy at a tertiary children's hospital from July 2007-July 2018. Exclusion criteria included known malignancy, immunologic deficiency, or high suspicion for congenital neck mass or acute bacterial lymphadenitis $(\mathrm{N}=20)$. The primary outcome was surgical biopsy. Benign lymphadenopathy was determined by pathologic or clinical diagnosis. Data were analyzed by t-tests or chi-squared as appropriate.

Results: Overall 109 cases were included, with a mean age of $6.2 \pm 4.5$ years, and presence for $10.3 \pm 12$ months prior to initial presentation. Seventeen (16\%) patients underwent surgical biopsy, all with benign pathology. In this group, lymphadenopathy was present for a shorter period ( $4.4 \pm 3.9 \mathrm{vs.} 11 \pm$ 13 months, $\mathrm{P}=0.03)$, and was larger on physical exam ( $2.1 \pm 1.1 \mathrm{vs.} 1.3 \pm 0.6 \mathrm{~cm}, \mathrm{P}<0.01)$.

Conclusion: The majority of pediatric cervical lymphadenopathy is benign and does not require surgical biopsy. Serial clinical follow-up with neck ultrasound can be used to safely monitor these children.
\end{abstract}

\section{INTRODUCTION}

Cervical lymphadenopathy is common in the pediatric population, with a prevalence ranging from 28-55\% [1,2]. Lymphoma, although rare, is one of the most common malignancies presenting in children with high cure rates when identified early [3]. When concerning features are present, such as B symptoms or supraclavicular lymphadenopathy, the decision to pursue further investigation is relatively straightforward, and several algorithms have been proposed to guide management $[4,5]$.

However, in cases of persistent but otherwise asymptomatic lymphadenopathy, individual practice patterns vary, and current literature does not provide evidence-based guidelines for safe and appropriate follow-up.

While prior studies have identified certain clinical, laboratory and radiographic features for suspicious lymphadenopathy, few have addressed the natural history and work-up required for patients who present with asymptomatic cervical lymphadenopathy. One recent prospective study which was aimed at describing the etiologies of all clinical presentations of cervical adenopathy, demonstrated that among 218 patients, the majority of lymphadenopathy resolved within 8 weeks and that those patients eventually diagnosed with malignancy either had B symptoms or abnormal hilar findings on ultrasound [6]. These data suggest that watchful waiting is a rational approach for asymptomatic cervical lymphadenopathy.

In this study, we sought to review our institutional experience of patients with asymptomatic, persistent lymphadenopathy with the hope of being able to provide guidelines for safe, non-invasive monitoring.

\section{METHODS}

A retrospective cohort study was performed to identify pediatric patients re- ferred to the University of California, San Francisco outpatient departments of Pediatric Otolaryngology and Pediatric Surgery. This study was approved by the Institutional Review Board of the University of California San Francisco.

\section{Study Group and Inclusion Criteria}

A retrospective cohort study was performed to identify pediatric patients less than 18 years of age with a diagnosis of cervical lymphadenopathy referred to an outpatient academic pediatric otolaryngology or general surgery clinic. Medical records were analyzed for initial outpatient evaluations between July 2007 and July 2018. This end date was selected to allow at least a 6-month follow-up period. A total of 133 patients were identified, and those with obvious neck infections or abscesses, congenital neck masses, or known rheumatologic, immunologic, or malignant conditions were excluded $(\mathrm{N}=20)$. In addition, 2 patients with high clinical suspicion for malignancy, 1 patient referred from pediatric rheumatology with diffuse lymphadenopathy and systemic symptoms, and 1 patient with high clinical suspicion for tuberculosis including overlying skin changes were excluded from all subsequent analyses.

\section{Clinical and Radiographic Data}

Clinical data extracted from charts included pertinent antecedent symptoms, medical history, and physical exam features including lymph node location, consistency, and overlying skin changes. Lymph node location were classified as follows: anterior cervical (defined as lymphadenopathy overlying the sternocleidomastoid or anterior to the muscle), posterior cervical (any lymph node posterior to the sternocleidomastoid), submandibular, submental, postauricular and supraclavicular. All lymphadenopathy dimensions, noted on either physical exam or ultrasound report, were reported using the largest dimension. If multiple lymph nodes were present, the largest lymph node was used. Ultrasound imaging reports were also reviewed for any commentary on nodal architecture or abnormal features. 
Table 1. Cohort Characteristics $(\mathrm{N}=109)$

\begin{tabular}{|c|c|}
\hline Characteristic & Mean $( \pm S D)$ or $N(\%)$ \\
\hline Age (years) & $6.2( \pm 4.5)$ \\
\hline Male & $57(53 \%)$ \\
\hline Time Since Mass Present (months) & $10.3( \pm 12)$ \\
\hline Size on Exam $(\mathrm{cm})$ & $1.5( \pm 0.7)$ \\
\hline Size on Ultrasound (cm) & $1.9( \pm 1.0)$ \\
\hline \multicolumn{2}{|l|}{ Location } \\
\hline Anterior & $40(39 \%)$ \\
\hline Posterior & $39(38 \%)$ \\
\hline Submandibular & $5(5 \%)$ \\
\hline Submental & $12(11 \%)$ \\
\hline Post-auricular & $5(5 \%)$ \\
\hline Supraclavicular & $2(2 \%)$ \\
\hline Initial Ultrasound & $67(61 \%)$ \\
\hline Follow-up Ultrasound & $17(25 \%)^{\star}$ \\
\hline Fine Needle Aspiration & $6(5.5 \%)$ \\
\hline Surgical Biopsy & $17(16 \%)$ \\
\hline
\end{tabular}

"The percentage is based on the 67 patients who underwent an initial ultrasound. SD, standard deviation.

Table 2. Comparison of Clinical and Radiographic Findings Between Group Undergoing Surgical Excision Versus No Surgical Excision

\begin{tabular}{|c|c|c|c|}
\hline \multicolumn{4}{|l|}{ Surgical Excision Status } \\
\hline Characteristic & No Surgical Excision $(\mathrm{N}=92)$ & Surgical Excision $(N=17)$ & P-Value \\
\hline Age & $6.3 \pm 4.5$ & $6.1 \pm 4.4$ & 0.92 \\
\hline Male & $50(54 \%)$ & $7(41 \%)$ & 0.32 \\
\hline Time Since Mass Was Present (months) & $11 \pm 13$ & $4.4 \pm 3.9$ & 0.03 \\
\hline Size on Exam & $1.3 \pm 0.6$ & $2.1 \pm 1.1$ & $<0.01$ \\
\hline Size on Ultrasound $(\mathrm{cm})$ & $1.8 \pm 0.9$ & $2.3 \pm 1.0$ & 0.11 \\
\hline Location & & & 0.31 \\
\hline Anterior & $36(42 \%)$ & $4(23 \%)$ & \\
\hline Posterior & $31(36 \%)$ & $8(47 \%)$ & \\
\hline Submental & $4(5 \%)$ & $1(6 \%)$ & \\
\hline Submandibular & $11(13 \%)$ & $1(6 \%)$ & \\
\hline Post-Auricular & $3(3 \%)$ & $2(12 \%)$ & \\
\hline Supraclavicular & $1(1 \%)$ & $1(6 \%)$ & \\
\hline
\end{tabular}

Data reported as mean \pm SD or N (\%). SD, standard deviation.

\section{Primary Outcome and Statistical Analysis}

The primary outcome was defined as surgical excision, including either incisional or excisional biopsy. All final pathology reports were abstracted from the medical record. Data were analyzed by chi squared or t-tests as appropriate (Stata 14.0, College Station, TX).

\section{RESULTS}

\section{Cohort Characteristics}

A total of 109 pediatric patients with asymptomatic, cervical lymphadenopathy were identified following referral to an academic Pediatric Otolaryngology or Pediatric Surgery department (Table 1). The average age was 6.2 years, with reported duration of lymphadenopathy 10.3 months prior to the initial referral visit. Overall, the average lymphadenopathy size on ultrasound was 1.9 $\mathrm{cm}$, while the average size on physical exam was $1.5 \mathrm{~cm}$. The most commonly reported locations of cervical lymphadenopathy were anterior and posterior (40\% and 39\%, respectively). Only 6/109 patients underwent a fine needle aspiration (FNA), and 3/6 ultimately still underwent definitive surgical excision. Of the 17 patients who underwent surgical excision, no new cases of malignancy were identified on pathology.

\section{Univariate Analysis}

On univariate analysis, there was no difference in age, gender, or lymphadenopathy location between the groups undergoing surgical excision and the group undergoing continued observation (Table 2). Those who underwent surgical excision did have larger size lymphadenopathy based on physical exam compared to those who did not undergo surgical excision $(2.1 \pm 1.0 \mathrm{~cm}$ versus $1.3 \pm 0.6 \mathrm{~cm}, \mathrm{P}<0.01$ ); this trend was similarly observed based on ultrasound measurements, but did not reach statistical significance $(2.3 \pm 1.0 \mathrm{~cm}$ 


\begin{tabular}{lll}
\hline Table 3. Overview of Ultrasound Data & & \\
\hline Surgical Excision Status & & Surgical Excision $(\mathbf{N}=17)$ \\
\hline Characteristic & No Surgical Excision $(\mathbf{N}=92)$ & $11 / 17$ \\
\hline First US & $56 / 92$ & $0 / 4$ \\
\hline \multicolumn{1}{|c|}{ Presence of Fatty Hilum } & $15 / 21$ & $7 / 11$ \\
\hline Suggestive of Reactive Lymphadenopathy & $51 / 56$ & $2 / 11$ \\
\hline Second US & $15 / 56$ & $1 / 1$ \\
\hline Presence of Fatty Hilum & $4 / 5$ & $2 / 2$ \\
\hline Suggestive of Reactive Lymphadenopathy & $15 / 15$ & $17 \pm 15$ \\
\hline Time Between US (days) & $254 \pm 193$ & $0.9 \pm 1.0$ \\
\hline Average Change in Size $(\mathrm{cm})$ & $-0.02 \pm 0.4$ & \\
\hline
\end{tabular}

Data reported as mean \pm SD or $\mathrm{n} / \mathrm{N}$. SD, standard deviation; US, Ultrasound.

\section{Table 4. Ultrasound Characteristics Among Patients Undergoing Surgical Excision}

\begin{tabular}{|c|c|c|c|}
\hline Patient & Size on US $(\mathrm{cm})$ & US Characteristics & Final Pathology \\
\hline 1 & 0.9 & May be reactive & Reactive follicular hyperplasia, no lymphoma \\
\hline 2 & 3.2 & Enlarged lymph nodes with thickened cortices & Reactive lymph node \\
\hline 3 & 1.7 & Likely reactive lymph node & Reactive follicular and interfollicular hyperplasia, no lymphoma \\
\hline 4 & 1.5 & Hypoechoic, may be sebaceous cyst or parotid gland cyst & Reactive follicular lymphoid hyperplasia, no lymphoma \\
\hline 5 & 2.5 & $\begin{array}{l}\text { Numerous lymph nodes without fatty hilum. Repeat US } 1 \text { week later } \\
\text { with normal architecture and fatty hilum }\end{array}$ & Reactive follicular and paracortical hyperplasia \\
\hline 6 & 4.5 & Enlarged lymph node; no necrosis or fluid collection & Reactive follicular lymphoid hyperplasia with focal architectural atypia \\
\hline 7 & 2.7 & $\begin{array}{l}\text { Multiple enlarged bilateral cervical lymph nodes, all with normal } \\
\text { morphology with fatty hilum, favored to be reactive; follow-up US } \\
\text { after } 1 \text { month stable }\end{array}$ & Reactive lymph node with follicular hyperplasia \\
\hline 8 & 2.0 & Submandibular lymphadenopathy & $\begin{array}{l}\text { Necrotizing granulomata with granulation tissue, special stain negative } \\
\text { for acid-fast bacilli }\end{array}$ \\
\hline 9 & 4.0 & $\begin{array}{l}\text { Small calcifications, most likely etiology would be granulomatous } \\
\text { disease }\end{array}$ & $\begin{array}{l}\text { Necrotizing granulomatous inflammation, mycobacterium tuberculosis } \\
\text { complex PCR positive }\end{array}$ \\
\hline 10 & 3.0 & $\begin{array}{l}\text { Fibrous hamartoma of infancy: lobulated, heterogeneous echotex- } \\
\text { ture, multiple intervening areas of hyperechogenicity, internal } \\
\text { vascularity with high flow }\end{array}$ & Vascular malformation consistent with arteriovenous malformation \\
\hline 11 & 1.4 & Ovoid hypoechoic circumscribed nodule consistent with lymph node & Neurofibroma \\
\hline 12 & 1.3 & Oval shaped, well-defined margins, likely reactive & Pilomatrixoma \\
\hline 13 & 1.3 & Heterogeneous well-circumscribed solid mass & Pilomatrixoma \\
\hline 14 & NA & US not performed & Reactive follicular hyperplasia, no lymphoma \\
\hline 15 & NA & US not performed & $\begin{array}{l}\text { Lymph node with intact architecture and reactive follicular hyperplasia; } \\
\text { flow cytometry negative }\end{array}$ \\
\hline 16 & NA & US not performed & Reactive follicular lymphoid hyperplasia with architectural atypia \\
\hline 17 & NA & US not performed & Necrotizing granulomatous infection with acid-fast bacilli \\
\hline
\end{tabular}

NA, Not Applicable; US, Ultrasound.

versus $1.8 \pm 0.9 \mathrm{~cm}, \mathrm{P}=0.11)$. The length of time the lymphadenopathy had been present prior to initial presentation was shorter in the surgical excision group compared to the group not undergoing surgery $(4.3 \pm 3.7$ months versus $11 \pm 13$ months, $P=0.02$ ).

\section{Ultrasound Characteristics}

Sixty-seven patients underwent an initial ultrasound. Further details regarding the use of ultrasound are found in Table 3. In both the surgical and no surgical excision groups, $61 \%$ of patients underwent at least 1 ultrasound. Among those who did not undergo biopsy, 51/56 had findings suggestive of reactive lymphadenopathy, compared to 7/11 in those who did undergo biopsy. The $5 / 56$ that were inconsistent with reactive lymphadenopathy had the following features: 2 with report of a non-specific hypoechoic mass, 1 with an abnormal solid mass that was no longer palpable at the time of presentation, 1 possi- ble infected ranula, and 1 consistent with fibromatosis coli. The remaining US details regarding patients undergoing surgical excision are found in Table 4. Overall, only $37 \%$ (25/67) of the initial ultrasound reports commented on the presence or absence of a fatty hilum. Follow-up ultrasound was uncommon with only 15/56 in the no surgical excision group and 2/11 in the surgical excision group. However, all the follow-up ultrasounds demonstrated reassuring radiographic characteristics.

\section{Detailed Overview of Patients Undergoing Surgical Excision}

Seventeen patients underwent surgical biopsy under general anesthesia, with pathology results shown in Figure 1 and the associated ultrasound characteristics are described in Table 4. Of the 17 patients, one had a post-operative course complicated by a surgical site abscess requiring multiple presentations to medical care and an additional surgical procedure for treatment. 


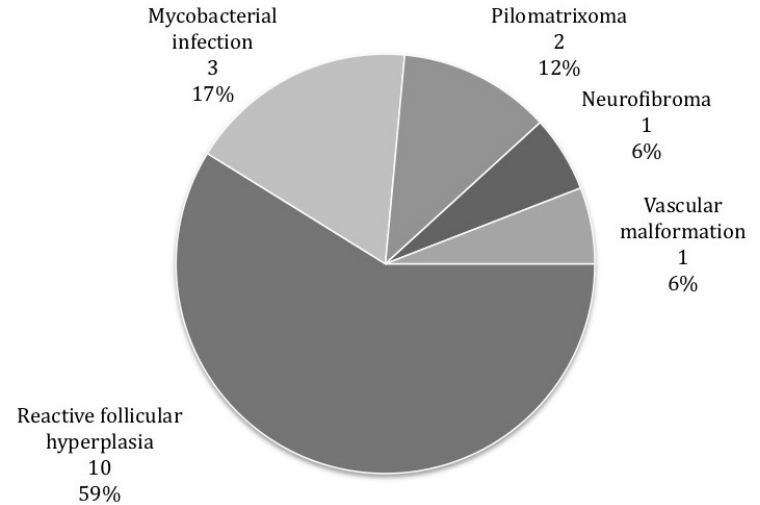

Figure 1. Surgical pathology results $(\mathrm{N}=17)$.

The most common pathologic diagnosis was reactive follicular hyperplasia (10/17, 59\%). Six of these patients underwent an ultrasound with findings consistent with benign, reactive lymphadenopathy; one had ultrasound findings concerning for a possible cyst; three did not get an ultrasound. Overall, 3 patients were diagnosed with atypical mycobacteria. One patient was clinically asymptomatic at the time of presentation, but a PPD order prior to the visit was positive; subsequent MRI showed findings consistent with atypical mycobacteria. One patient had no symptoms, but had a history of isoniazid treatment, and US revealed microcalcifications concerning for granulomatous disease.

Lastly, one had a left-sided cervical lymphadenopathy without other concurrent symptoms; prior to initial consultation, a PPD was placed which was positive, with a negative chest x-ray. Among the 11 patients who underwent an ultrasound, only 2 cases had discordant ultrasound features and final pathologic diagnosis.

\section{DISCUSSION}

Asymptomatic cervical lymphadenopathy in pediatric patients is a common outpatient referral diagnosis, yet no clear algorithm is uniformly currently accepted in defining criteria for surgical biopsy. In this retrospective study of 109 patients, one of the largest reported to date, we found that those with larger lymph nodes identified on clinical exam for a shorter time period were more likely to undergo surgical excision. Our most significant finding, however, is that all 17 patients undergoing surgical excision had benign pathology. This is the first study to date that describes the natural course of persistent, asymptomatic pediatric cervical lymphadenopathy.

The overall rate of malignancy among pediatric cervical lymphadenopathy is low. A prospective cohort study by Bozlak and colleagues described a rate of $2.7 \%$ [6]. This differs from our study in which there were no cases of newly diagnosed malignancy. This likely reflects the strict exclusion criteria that was used for our study population, as our aim was to describe asymptomatic, pediatric cervical lymphadenopathy. Additionally, while the incidence of new diagnosis of malignancy is low, the majority of pediatric malignant cases present with high clinical suspicion, even prior to imaging or surgical biopsy for confirmation [6-9]. This suggests that in asymptomatic clinical presentations, surgical biopsy may be deferred, and these findings are consistent with our study.

Prior literature has examined the utility of FNA as an alternative to open surgical biopsy for pediatric patients, as it does not always require general an- esthesia. However, in a retrospective study involving >200 patients 0-21 years old, 73\% still required sedation or general anesthesia [10]. Furthermore, even among patients who undergo FNA, false negative or inconclusive results are substantial, up to $28 \%$, and thus still requires excisional biopsy for pathologic confirmation[11,12]. These results are in agreement with our study given that even among the few patients who underwent an FNA, 50\% still underwent surgical excision.

Multiple studies have aimed to describe clinical and/or radiographic features that warrant surgical intervention. Prior studies have suggested that large cervical lymphadenopathy increases the suspicion for malignancy and/or need for excision. Bozlak et. al. found cervical lymphadenopathy $>3$ $\mathrm{cm}$ was more likely to be malignant than those $<3 \mathrm{~cm}$. This differs from our study as even among those with larger lymphadenopathy, none were diagnosed with malignancy. This difference is likely because our study focused on patients with asymptomatic lymphadenopathy, thus patients with additional peripheral lymphadenopathy or B symptoms at presentation were excluded [6]. A retrospective cohort study of 50 hospitalized pediatric patients with cervical lymphadenopathy found that abnormal ultrasound characteristics, specifically using the long to short axis and the intranodal vascularity, may help guide the decision for surgical excision [13]. Although our study did not standardize the collection of ultrasound measurements and architecture, we did note that ultrasound findings were often concordant with final pathology; together these data support that ultrasonography remains a useful, low-risk option to help risk-stratify patients with cervical lymphadenopathy. Using ultrasound for assistance with diagnosis remains an area of interest [14], with many studies suggesting it as first-line imaging for pediatric lymphadenopathy [15]. Niedzieleska et al. demonstrated that abnormal shape of a lymph node and/or hilum or abnormal intranodal vascularization on ultrasound suggests etiologies besides benign/reactive lymphadenopathy and warrant further investigation [16]. They diagnosed two new cases of malignancy; one case had a concerning ultrasound, with hypoechoic background and abnormal hilum, and the second case presented with clinical symptoms including stomatitis and gingival pain. This study is consistent with our findings that surgical biopsy can often be spared in asymptomatic patients with a reassuring ultrasound.

There were potential limitations to our study. Our analysis was based on retrospective data collection, and thus subject to missing information. In particular, the ultrasound data that we reported were collected from the imaging reports, rather than a review of the raw images. Thus, details such as nodal architecture or size, which are important to assist with risk stratifying lymphadenopathy, were not uniformly reported or missing. However, even with some limited ultrasound information, we were still able to conclude that concordance between ultrasound characteristics and final pathology is high. Additionally, our study analyzed children referred to a tertiary care institution, and the number of patients undergoing surgical excision is small. Therefore, our results may not be generalizable to broader populations. Further, while patients had at least a 6-month follow-up period which is likely sufficient based on prior studies [6], extrapolation of longer-term follow-up guidelines are limited. Finally, while this study represents the largest known group of asymptomatic pediatric cervical lymphadenopathy patients, more robust multivariate analysis was still limited by the sample size, and additional differences in clinical or radiographic features may not have been detected.

\section{CONCLUSION}

For pediatric patients with asymptomatic cervical lymphadenopathy, diagnosis of malignancy remains rare. Our findings suggest that even for patients with long-standing, palpable lymphadenopathy, observation with serial ultrasounds is reasonable. This may help to alleviate parental anxiety that may guide clinical decision-making for definitive excision and avoid the risks of unnecessary general anesthesia and surgical biopsy. 


\section{ARTICLE INFORMATION}

\section{Katherine C. Wai and TammyJ. Wang contributed equally to this work.}

*Correspondence: Katherine C. Wai, MD. Department of Otolaryngology-Head and Neck Surgery, University of California San Francisco, $55016^{\text {th }}$ St, Box 3213, San Francisco, CA, 94158, USA. Email: katherine.wai@ucsf.edu

Received: Sept. 24, 2019; Accepted: Nov. 25, 2019; Published:Jan. 06, 2020

DOI: $10.24983 /$ scitemed.aohns.2020.00121

Ethics Approval and Consent to Participate: The study is in accordance with the ethical standards of the 1964 Helsinki declaration and its later amendments or comparable ethical standards.

Funding: The study did not receive any specific grant from funding agencies in the public, commercial, or not-for-profit sectors.

Conflict of Interest: The authors report no financial or other conflict of interest relevant to this article, which is the intellectual property of the authors.

Copyright (c) 2020 The Authors. This is an open-access article distributed under the terms of the Creative Commons Attribution 4.0 International License (CC-BY).

\section{ACKNOWLEDGMENTS}

Author Contributions: KCW, TJW and KWR had full access to all the data in the study and take responsibility for the integrity of the data and the accuracy of the data analysis.

Study Concept and Design: All authors

Acquisition and Interpretation of Data: All authors

Drafting of Manuscript: KCW, TJW

Critical Revision of Manuscript for Important Intellectual Content: All authors

Statistical Analysis: KCW

Study Supervision: KWR

\section{DISCLOSURES}

This work was presented as a podium presentation at the American Society of Pediatric Otolaryngology Summer meeting on July 21, 2019 in Vail, Colorado, USA.

\section{REFERENCES}

1. Larsson LO, Bentzon MW, Berg Kelly K, et al. Palpable lymph nodes of the neck in swedish schoolchildren. Acta Paediatr 1994;83(10):1091-1094.

2. Park YW. Evaluation of neck masses in children. Am Fam Physician 1995;51(8):1904 1912.

3. Buhtoiarov IN. Pediatric lymphoma. Pediatr Rev 2017;38(9):410-423.

4. Rosenberg TL, Nolder AR. Pediatric cervical lymphadenopathy. Otolaryngol Clin North Am 2014;47(5):721-731.

5. Chiappini E, Camaioni A, Benazzo M, et al. Development of an algorithm for the management of cervical lymphadenopathy in children: Consensus of the italian society of preventive and social pediatrics, jointly with the italian society of pediatric infectious diseases and the italian society of pediatric otorhinolaryngology. Expert Rev Anti Infect Ther 2015;13(12):1557-1567.

6. Bozlak S, Varkal MA, Yildiz I, et al. Cervical lymphadenopathies in children: A prospective clinical cohort study. Int J Pediatr Otorhinolaryngol 2016;82:81-87.

7. Citak EC, Koku N, Demirci M, Tanyeri B, Deniz H. A retrospective chart review of evaluation of the cervical lymphadenopathies in children. Auris Nasus Larynx 2011;38(5):618-621.

8. Locke $\mathrm{R} C \mathrm{R}$, Kubba $\mathrm{H}$. When does an enlarged cervical lymph node in a child need excision? A systematic review. Int J Pediatr Otorhinolaryngol 2014;78(3):393-401.

9. Riva G SM, Peradotto F, Scolfaro C, Di Rosa G, Tavormina P. Pediatric neck masses: How clinical and radiological features can drive diagnosis. Eur J Pediatr 2019;178(4):463-471

10. Huyett $\mathrm{P}$, Monaco SE, Choi SS, Simons JP. Utility of fine-needle aspiration biopsy in the evaluation of pediatric head and neck masses. Otolaryngol Head Neck Surg 2016;154(5):928-935.

11. Anne S, Teot LA, Mandell DL. Fine needle aspiration biopsy: Role in diagnosis of pediatric head and neck masses. Int J Pediatr Otorhinolaryngol 2008;72(10):15471553.

12. Alam $K$, Khan $R$, Jain $A$, et al. The value of fine-needle aspiration cytology in the evaluation of pediatric head and neck tumors. Int J Pediatr Otorhinolaryngol 2009;73(7):923-927.

13. Papadopouli E, Michailidi E, Papadopoulou E, Paspalaki P, Vlahakis I, Kalmanti M Cervical lymphadenopathy in childhood epidemiology and management. Pediatr Hematol Oncol 2009;26(6):454-460.

14. Restrepo R, Oneto J, Lopez K, Kukreja K. Head and neck lymph nodes in children: The spectrum from normal to abnormal. Pediatr Radio/ 2009;39(8):836-846.

15. Weinstock MS, Patel NA, Smith LP. Pediatric cervical lymphadenopathy. Pediatr Rev 2018;39(9):433-443.

16. Niedzielska G, Kotowski M, Niedzielski A, Dybiec E, Wieczorek P. Cervical lymphadenopathy in children--incidence and diagnostic management. Int J Pediatr Otorhinolaryngol 2007;71(1):51-56. 